\title{
Metaheuristics algorithms to identify nonlinear Hammerstein model: a decade survey
}

\author{
Julakha Jahan Jui, Mohd Ashraf Ahmad, Muhammad Ikram Mohd Rashid \\ Faculty of Electrical and Electronics Engineering Technology, University Malaysia Pahang, Pekan, Pahang, Malaysia
}

\begin{abstract}
Article Info
Article history:

Received Jun 23, 2021

Revised Oct 26, 2021

Accepted Dec 26, 2021

Keywords:

Block oriented models

Hammerstein model

Metaheuristics

Nonlinear system identification

Optimization

Population-based optimization

ABSTRACT

Metaheuristics have been acknowledged as an effective solution for many difficult issues related to optimization. The metaheuristics, especially swarm's intelligence and evolutionary computing algorithms, have gained popularity within a short time over the past two decades. Various metaheuristics algorithms are being introduced on an annual basis and applications that are more new are gradually being discovered. This paper presents a survey for the years 2011-2021 on multiple metaheuristics algorithms, particularly swarm and evolutionary algorithms, to identify a nonlinear block-oriented model called the Hammerstein model, mainly because such model has garnered much interest amidst researchers to identify nonlinear systems. Besides introducing a complete survey on the various population-based algorithms to identify the Hammerstein model, this paper also investigated some empirically verified actual process plants results. As such, this article serves as a guideline on the fundamentals of identifying nonlinear block-oriented models for new practitioners, apart from presenting a comprehensive summary of cutting-edge trends within the context of this topic area.
\end{abstract}

This is an open access article under the CC BY-SA license.



\section{Corresponding Author:}

Mohd Ashraf Ahmad

Faculty of Electrical and Electronics Engineering Technology, University Malaysia Pahang

26600, Pekan, Pahang, Malaysia

Email: wichian.sit@mfu.ac.th

\section{INTRODUCTION}

System identification is vital in estimating a model that can mimic the attributes of a system [1]. Since past years, the identification and estimation of parameters for both nonlinear and linear systems have been vastly investigated by a range of researchers. Since most practical systems are nonlinear in nature, it has become more popular in recent times. The block-oriented models within the nonlinear system have been reckoned and mostly applied for automatic control and system detection. These block-oriented models are composed of a range of interconnections between static nonlinear and linear dynamic components. The three simple and widely-applied nonlinear block-oriented models to model practical systems refer to Hammerstein models and Wiener models, as well as the combination of both models [2].

In a traditional method of system identification, the reason for applying gradient-based algorithms for estimating parameters in a model is because it minimizes the mean square error (MSE) for both model and system [3]. For instance, an iterative algorithm based on a gradient descent algorithm was employed to identify the Hammerstein model [4]. In identifying the Wiener model and H-W model, Wang et al. [5] proposed gradient descent based and least square based iterative algorithm. The most generally used algorithm amongst all adaptive-type algorithms refers to least means square (LMS) [6]. In most cases of system identification, the error surface is multimodal and demands convergence into a sub-optimal 
alternative by using the standard strategy of gradient descent [7]. This limitation can be overcome by using varied stochastic search approaches, such as the swarm intelligence based and evolutionary computing-based optimisation algorithms.

Swarm intelligence algorithms refer to an efficient distributed intelligence framework for solving optimisation intricacies, which has been inspired by the mutual behaviour displayed by colonies of social insects and societies of other animals. Some optimisation algorithms that derived from the swarming behaviour metaphor are ant colony optimisation [8], particle swarm optimisation (PSO) [9], bacterial foraging optimisation (BFO) [10] and bee colony optimisation [11]. Evolutionary computation is the generic word over several optimisation algorithms influenced by the Darwinian principles of the power of nature to develop well-adapted living beings to their surroundings. The fields of genetic algorithm (GA) [12], evolutionary programming [13], and genetic programming [14] fall under the evolutionary computing algorithms, also known as evolutionary algorithms. The differential evolution algorithm (DEA) is also widely applied to address ongoing issues of global optimisation [15] and appeared to be a quite accurate optimisation technique for many distinct tasks. Many studies have also reported using such a type of optimisation algorithm to identify both linear and nonlinear systems.

The pie chart in Figure 1 presents an overview of some popular block-oriented models used to identify nonlinear systems through the implementation of various metaheuristics algorithms. The pie chart is composed of three fractions that depict their usage popularity in identifying nonlinear systems; Hammerstein model leading with $53 \%$ (blue), followed by Wiener model with $32 \%$ (red), and the H-W model with $15 \%$ usage (green). It appears that most research favoured the Hammerstein model to identify nonlinear systems with a range of metaheuristics algorithms.

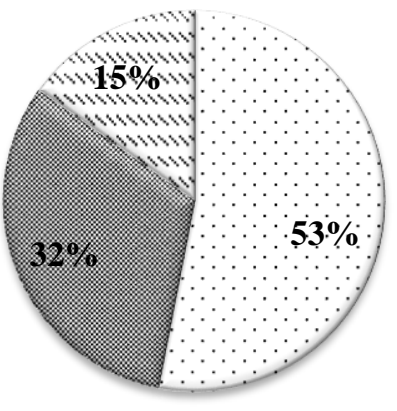

• Hammerstein model

Wiener model

SHammerstein - Wiener

model

Figure 1. Pie chart of block-oriented models popularity using metaheuristics algorithms

The bar chart in Figure 2 displays the relationships between different types of metaheuristics algorithms and their frequency of use for parameter estimation using block-oriented models. The metaheuristics algorithms are GA, PSO, DEA, glow-worm swarm optimisation (GSO), ant colony optimisation (ACO), BFO, colliding bodies optimisation (CBO), and brain storm optimisation (BSO). In the case of GA, Hammerstein and H-W models were used eight times each, while seven times for the wiener model. In PSO, Hammerstein, Wiener, and H-W models were used 17, 14, and three times, respectively. As for DE, Hammerstein, Wiener, and H-W models were used four, nine, and two times, respectively. Other metaheuristics algorithms were quite unfamiliar in identifying block-oriented models, as depicted in the bar chart. The bar chart shows that among all the metaheuristics; GA, PSO, and DE were mostly applied for parameter estimation. Thus, an extensive analysis is elaborated in this paper using three distinct optimisation methods (GA, PSO, and DE) to effectively identify the Hammerstein model.

In this paper, we give an overview of the nonlinear Hammerstein system identification using several metaheuristics. Since the traditional methods have various limitations such as the creation of multimodal error surface, complexity in cost function minimization and inability to converse in global optima, the metaheuristics algorithms could be a good alternative for applying in nonlinear Hammerstein system identification. Metaheuristics can balance exploration and exploitation, it requires very few control parameters which will help to reduce the computational complexity and it has also the ability to converge in global optima. On the other hand, the Hammerstein model identification has been a hot research field because it can be successfully applied for the identification of real experimental plants and processes. Therefore, this paper could be provided a good research direction for the new researchers in the identification of nonlinear Hammerstein systems.

The remaining part of the paper is as; section 2 discusses the general diagram of the Hammerstein

Metaheuristics algorithms to identify nonlinear Hammerstein model: a decade survey (Julakha Jahan Jui) 
model and the review of the use of evolutionary and swarm optimisation algorithms to identify the Hammerstein model. Empirical verification of varied plants and processes is given in section 3, while section 4 depicts future research trends in this area. Lastly, section 5 concludes this study.

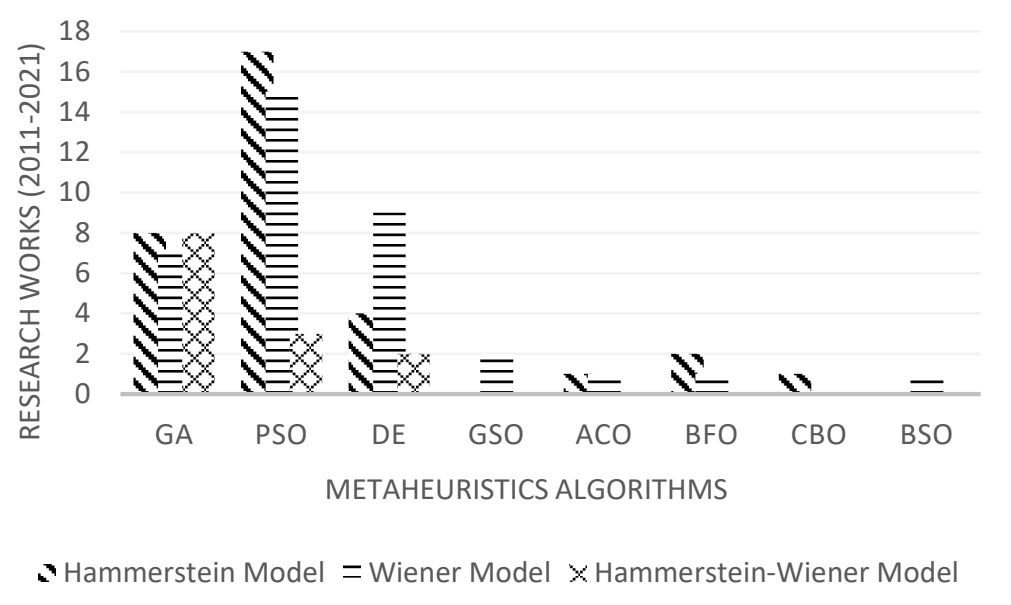

Figure 2. Bar graph: block-oriented models identification algorithms based on popularity

\section{RELATED WORK}

Scientific or information-based modelling, commonly known as system identification, is a significant aspect in the science and engineering domain. Comprehensive system identification development based on a linear model is vast and the related theories are mature. Researchers worldwide have been always interested in identifying both nonlinear and dynamic systems. For any control system design, system identification is the main component, and it is challenging to identify nonlinear systems in the present time. The nonlinear block-oriented systems, namely Hammerstein, Wiener, and H-W models, are interesting research areas amongst the academic and industrial environments. Among the three the Hammerstein model is the popular one which we can see from the pie chart in Figure 1. This section presents an overview of the block-oriented nonlinear Hammerstein model along with its block diagrams.

The linear function in the Hammerstein model is preceded by nonlinear block, as shown in Figure 3, where $u(t)$ is training input, $x(t)$ denotes nonlinear system output, $y^{\prime}(t)$ represents Hammerstein model output, and $v(t)$ signifies noise. The linear/nonlinear parameters are modified using metaheuristics algorithm for Hammerstein identification system, mainly to reduce cost function, for instance, MSE and least means square error (LMSE). From Figure 3, the nonlinear static block output is:

$$
x(t)=f(u(t))
$$

and $y(t)$ is labelled as $y^{\prime}(t)$ due to disruption by noise $v(t)$. The input-output correlation is:

$$
y^{\prime}(t)=H(s) x(t)+v(t)
$$

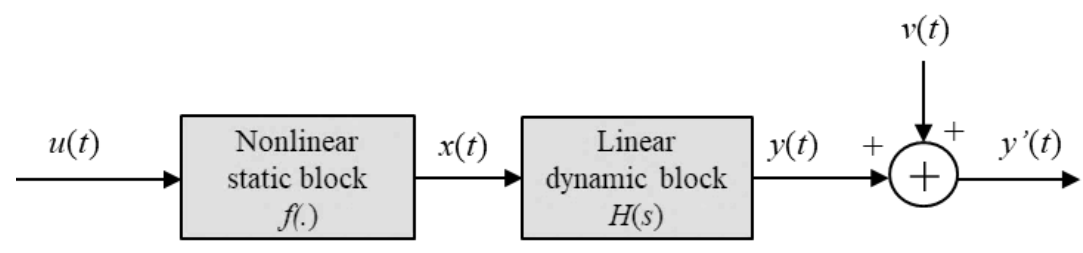

Figure 3. Block diagram of block-oriented nonlinear Hammerstein model

Figure 4 shows the conceptual structure of nonlinear system identification based on the Hammerstein model. Error minimization was determined to assess its objective function using (3): 


$$
J=\int_{0}^{n t_{s}}\left(y^{\prime}(t)-\hat{y}(t)\right)^{2} d t
$$

where $J$ is defined as the objective function, $y^{\prime}(t)$ and $\hat{y}(t)$ are the real and estimated outputs, whereas $t_{s}$ is sampling time. The optimisation tool (algorithm) to update the Hammerstein model at the next iteration uses the variance between real and estimated outputs. This tuning step is iteratively performed until the last iteration.

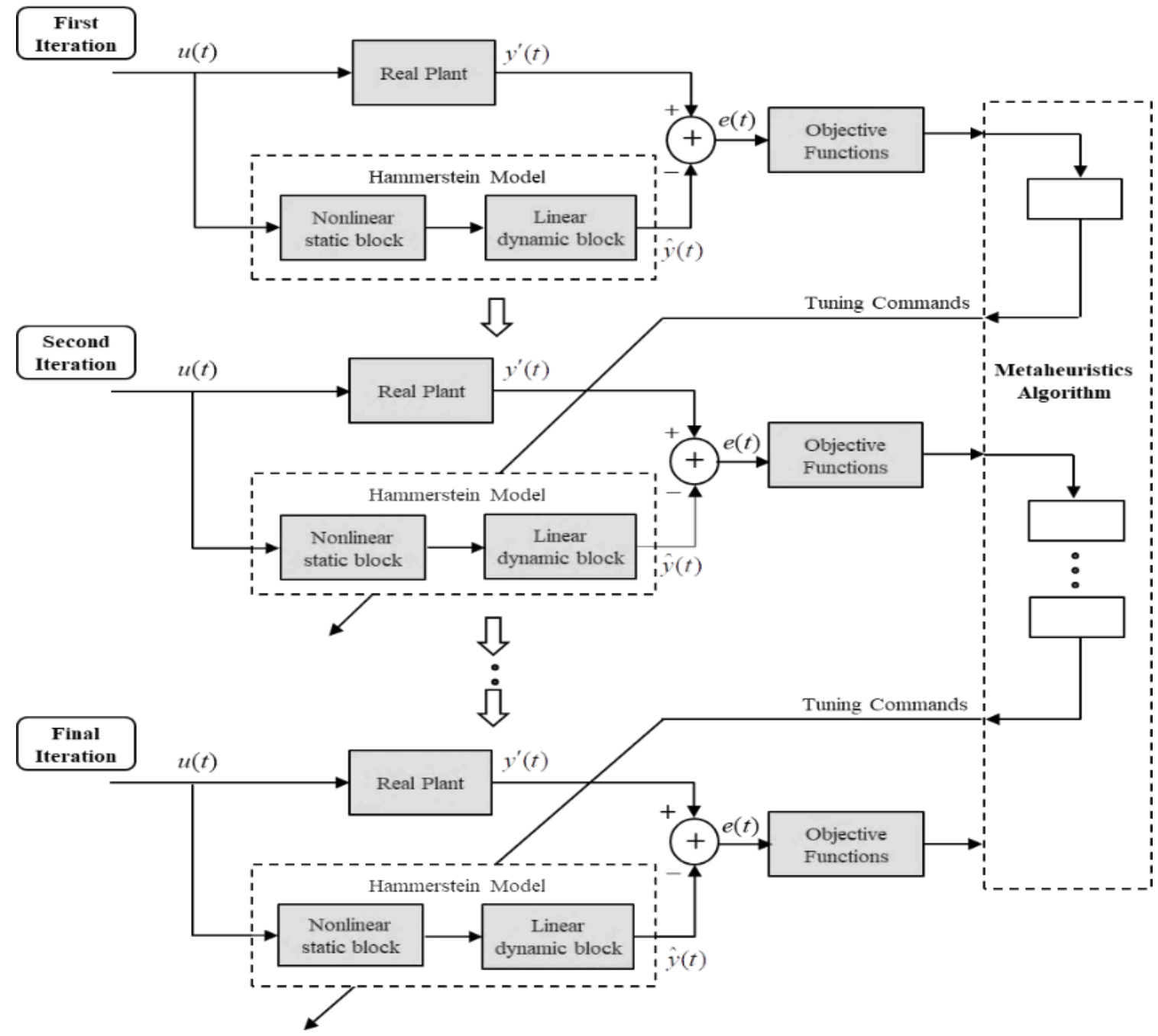

Figure 4. Algorithm tuning process using the Hammerstein model

This section also explains the Hammerstein model that is widely used to identify real nonlinear plants and processes [16], [17], including Thermoelectric cooler [18], [19], amplified piezoelectric tube actuators [20], forecast of wind speed [21], giant magnetostrictive actuators (GMAs) [22], boiler superheated steam pressure [23], boost converter of DC/DC [24], intelligent pneumatic actuator (IPA) [25], hydraulic mini excavator [26], ultrasonic motor [27], proton exchange membrane fuel cell (PEMFC) [28], turntable servo system [29], valve Stiction [30] and electrically stimulated muscle [31]. The Hammerstein model can accurately model actuators to solve control issues and strengthen nonlinearity system identification. This section reviews the Hammerstein identification scheme using soft and evolutionary computing methods. The two Hammerstein identification schemes are parametric and nonparametric. Nonlinearity is polynomial in parametric recognition system but modelled with probabilistic methods for the non-parametric system. The methods of Hammerstein identification are stochastic gradient method [32], least squares method (LSM) [33], subspace method [34], iterative method [35], as well as swarm and evolutionary algorithms.

Akramizadeh et al. [36] initially proposed the GA for the identification of the Hammerstein system. They used GA for determining the correct nonlinear function structure and parameters, as well as the number 
of zeros and poles of the linear transfer function. Lai et al. [37], the piezoceramic actuator is identified by the Hammerstein-based model and the parameters are identified using GA. They were used the Hammersteinbased model for the piezoceramic actuator for describing the frequency dependency. In 2015, Li et al. [38] proposed hybrid GA for the identification of the Hammerstein model and shows that hybrid GA has strong efficacy and robustness, has a good recognition effect, and is a viable solution for solving the nonlinear recognition problem. Qian et al. [29] identify the turntable servo system by using the Hammerstein model. They used GA to optimize the global control variable by minimizing the cost function, but their limitation is that the system properties differ in different working states. A nonlinear dynamic process called Blast furnace (BF) in ironmaking is identified and controlled in [39] where they used the Hammerstein model for the prediction of most essential quality (MIQ) indices. They employed the GA-based nonlinear model predictive control (GA-NMPC) method to demonstrate the applicability of the Hammerstein model for control design. Another plant called proton exchange membrane fuel cell (PEMFC) is modelled in [28] based on the fractional Hammerstein model and a fuzzy GA is adopted to acquire the best fractional order of the Hammerstein system. Next, a nonlinear multi-input single-output (MISO) system identification method using Hammerstein model was presented in [40], which involved fractional transfer function and modified radial base function NN (MRBFNN) as the linear dynamic component and a static nonlinear subsystem, in which modified GA defined the fractional orders, as well as the width and centre of MRBFNN, apart from identifying the initial prediction of other unidentified parameters. Genetic algorithms (GAs) are used to identify parameters in a nonlinear Hammerstein controlled autoregressive (NHCAR) system but the optimization process necessitates additional execution time, generation counts, and function counts [41]. A similar approach is used for identifying the continuous-time Hammerstein system in [42]. In the paper they hybrid the GA with the recursive least-squares (RLS) method and their proposed method shows a superior identification performance. In another study, the Hammerstein controlled auto regressive auto regressive moving average (HCARARMA) system is proposed and the parameter is estimated by using DE, GAs, pattern search (PS) and simulated annealing (SA) algorithms [43]. They show that the complexity measures for GAs are higher than the rest of the optimization mechanisms. Similarly, a nonlinear Hammerstein controlled auto regressive auto regressive (NHCARAR) system is proposed in [44] and the parameters are identified by using a backtracking search algorithm (BSA), DE and GAs. In the study, it was found that the BSA and DE algorithms outperformed the GAs, with BSA providing equivalent accuracy at the expense of greater complexity than DE.

PSO and its variants are the most popular identification method for the Hammerstein system. Using PSO, parameter estimation, and model selection was performed for the Hammerstein model. A method to quantify valve stiction in control loops using PSO was reported in [30]. The PSO from input-output data were used to estimate the parameters of the Hammerstein model by decreasing the errors between real and identified model outputs. The immunized PSO, which exerted more CPU load, was found to enhance modelling precision more than PSO did, while the clonal PSO gave PSO-like modelling precision and CPU load. Enhanced PSO gave better output than conventional PSO did [45]. For iterations of a MISO Hammerstein system identification task, the improved PSO (IPSO) gave more rapid convergence and better accuracy than the conventional PSO did, while neglecting runtime iteration. A work comparable to [45] was presented in [46], with the distinction that in place of mutation, the IPSO contained dynamically reduced inertia. To depict nonlinear block, the Hammerstein model was applied with non-uniform rational NN Bspline (NURB) [47]. NN shaping parameters were estimated by PSO and the other parameters using the overparameterization method [48], but the evolutionary algorithms used for training were unreported. Upon using both maximum likelihood adaptive PSO (ML-APSO) and maximum likelihood PSO (ML-PSO) for the Hammerstein model, ML-APSO gave better convergence speed and modelling precision than ML-PSO and recursive least square (RLS) algorithm did [49]. Although convergence speed and efficiency were achieved by RLS-PSO, no comparison was made with PSO and RLS [50]. The CBO algorithm, inspired by the concept of a collision between bodies [51], was implemented in [52] for Hammerstein system modelling. Similarly, the performance displayed by CBO in [53] was better in terms of CPU time and MSE when compared with outcomes retrieved from CSA [54]. The Hammerstein model, along with other systems, were identified using a modified fish swarm algorithm (MFSA) [55], whereby MFSA displayed superior efficiency to GA, FSA, and PSO algorithms, while dismissing the convergence features of the algorithms. A similar algorithm was used to model a nonlinear dynamic system [56]. The CSA-based Hammerstein model was applied with FLANN and adaptive IIR filter modelling nonlinear and linear blocks, respectively [54]. As a result, the performance exhibited by CSA was superior to DE and PSO considering CPU time and lower MSE. Next, the Hammerstein model with GA managed to further decrease CPU time and MSE, when compared to GA alone, DE, and PSO [57]. Upon comparing two models of Hammerstein trained using DE, CSA, RLS, and GA; MPN with finite impulse response (FIR) filter (MPN-FIR) and volterra second-order with FIR (SOV-FIR), DE offered the best outcomes [58]. Using the Hammerstein model, the PSO and 
Weierstrass estimation theorems were applied to estimate the parameters and optimize the nonlinear system [59]. By comparing the modeling performance between the stochastic gradient algorithm and the modified method, PSO had improved the modeling accuracy over other comparative methods. A discrete-time fractional-order Hammerstein model is identified using the PSO algorithm [60]. In the presence of noise, their simulations confirm the efficiency of the method, but the result's accuracy decreases with a large amount of noise. Another proposed version of PSO is called the craziness based particle swarm optimization (CRPSO) algorithm for solving the local optima problem of PSO [61]. They apply their CRPSO algorithm for the identification of the discrete-time Hammerstein model. An adaptive particle swarm optimization algorithm (APSO) was proposed to identify the single input single output (SISO) Hammerstein system [62]. To improve the convergence speed of the conventional PSO, they applied an evolutionary state estimation technique. A new algorithm based on the mixed-integer particle swarm optimization (IR-PSO) for the estimation of the integer orders and the real coefficients of the block-oriented nonlinear Hammerstein autoregressive with exogenous input (NLHARX) system was proposed in [63]. Although PSO has gained attention from these researchers due to its searching capability, as well as its novelty, PSO can easily suffer from local optima stagnation when handling optimization problems with multiple local optima. Additionally, proper parameter settings will greatly influence the searching performance of PSO. Hence, the local optima entrapment and premature convergence of PSO often occur in numerous engineering optimization problems [64].

Xiong et al. initially introduced DE for the Hammerstein model to identify the position control in the ultrasonic motor [65]. The DE algorithm is used in this context to identify both the orders and parameters of the model. A study proposed the adaptive mutation DE (MDE) to estimate the parameters in the Hammerstein model. In contrast, another study optimized the Hammerstein model using DE to identify the system [66]. The MPN and the FIR models were used for nonlinear and linear elements, respectively. But they claim that, in terms of run times by DE, another algorithm has not always been very successful. This is a disadvantage of DE. Many studies considered that the modelling of polynomial nonlinearity is like that of a real one, which is uncertain in practical scenarios. Thus, determining the optimum extent of the polynomial nonlinearity is more practical. Next, the Hammerstein model with GA managed to further decrease the CPU time and MSE, when compared to GA alone, DE, and PSO [57]. Upon comparing the two models of the trained Hammerstein using DE, CSA, RLS, and GA, MPN with finite impulse response (FIR) filter (MPNFIR) and volterra second-order with FIR (SOV-FIR), DE offered the best outcomes [58]. In the study, it was found that DE required fewer control parameters compared to a well-known method, particle swarm optimization (PSO). These control parameters included population size, step size, and crossover rate. Furthermore, the study made by Vesterstrom and Thomsen [67] claimed that DE is better than PSO and EAs as it can obtain the lowest fitness solution for most of the problems reported in the literature. It was again reported that the convergence speed of DE is significantly faster than GA [58]. Additionally, DE is robust as it can consistently reproduce the same solutions over many simulations compared to PSO. This nonlinear USM of the Hammerstein model was introduced to properly categorize a nonlinear motor [27]. To improve both efficiency and performance of the model, a combination of the DEA and Hammerstein model was prescribed. A nonlinear Hammerstein controlled autoregressive (NHCAR) system was proposed, where the DE and GA were used for parameter estimation [44]. De also used to identify the Hammerstein system in [68], where they represent a real plant called electrically stimulated muscle (ESM) by using the Hammerstein structure. They claim that the DE method outperforms other metaheuristics such as GAS, PSO, pattern search (PS) and simulated annealing (SA). Recently, another real plant (ultrasonic motor) is modelled with the Hammerstein system and the DE algorithm is used to identify the model parameters [69]. A timeline of studies (2011-2021) involving the Hammerstein model incorporating evolutionary computing and swarm algorithms is given in Figure 5.

A few other metaheuristics algorithms also reported the identification of the Hammerstein system. The cuckoo search algorithm (CSA) based Hammerstein model was applied with FLANN and adaptive IIR filter modelling in nonlinear and linear blocks, respectively [54]. As a result, the performance exhibited by CSA was superior to DE and PSO considering the CPU time and lower MSE. The colliding bodies optimization (CBO) algorithm, inspired by the concept of a collision between bodies, was implemented for the Hammerstein system modelling [52]. The CBO gave better modelling precision and less CPU time than bacterial foraging optimization and adaptive PSO. Similarly, the performance displayed by CBO [53] was better in terms of CPU time and MSE when compared with outcomes retrieved from CSA [54], PSO, GA, and RLS, regardless of the colourful noise. A new meta-heuristic orthogonal colliding bodies optimization (OCBO) was proposed for the identification of the Hammerstein plant [70]. However, the drawback was the computational time of their proposed OCBO model is higher. Another metaheuristic called gravitational search algorithm (GSA) is proposed by Erik et al. in [71] for the identification of the ANFIS-Hammerstein model. They claim that the GSA algorithm is outperformed by the PSO and DE by avoiding critical flaws such as premature convergence to sub-optimal solutions. In another study, novel hybrid metaheuristics called average multi-verse optimizer and sine cosine algorithm (AMVO-SCA) is proposed for the identification of

Metaheuristics algorithms to identify nonlinear Hammerstein model: a decade survey (Julakha Jahan Jui) 
the continuous-time Hammerstein model [72]. They showed that their proposed method provides good identification accuracy than several well-known metaheuristics such as PSO, GWO, MVO and SCA.

$>$ Maximum Likelihood PSO (ML-PSO)

$>$ Maximum Likelihood Adaptive PSO (MLPSO),

$>$ Adaptive Mutation Differential Evolution Algorithm (MDE)

$>$ Recursive Least Squares PSO (RLS-PSO)

$>$ Colliding Bodies Optimization (CBO)



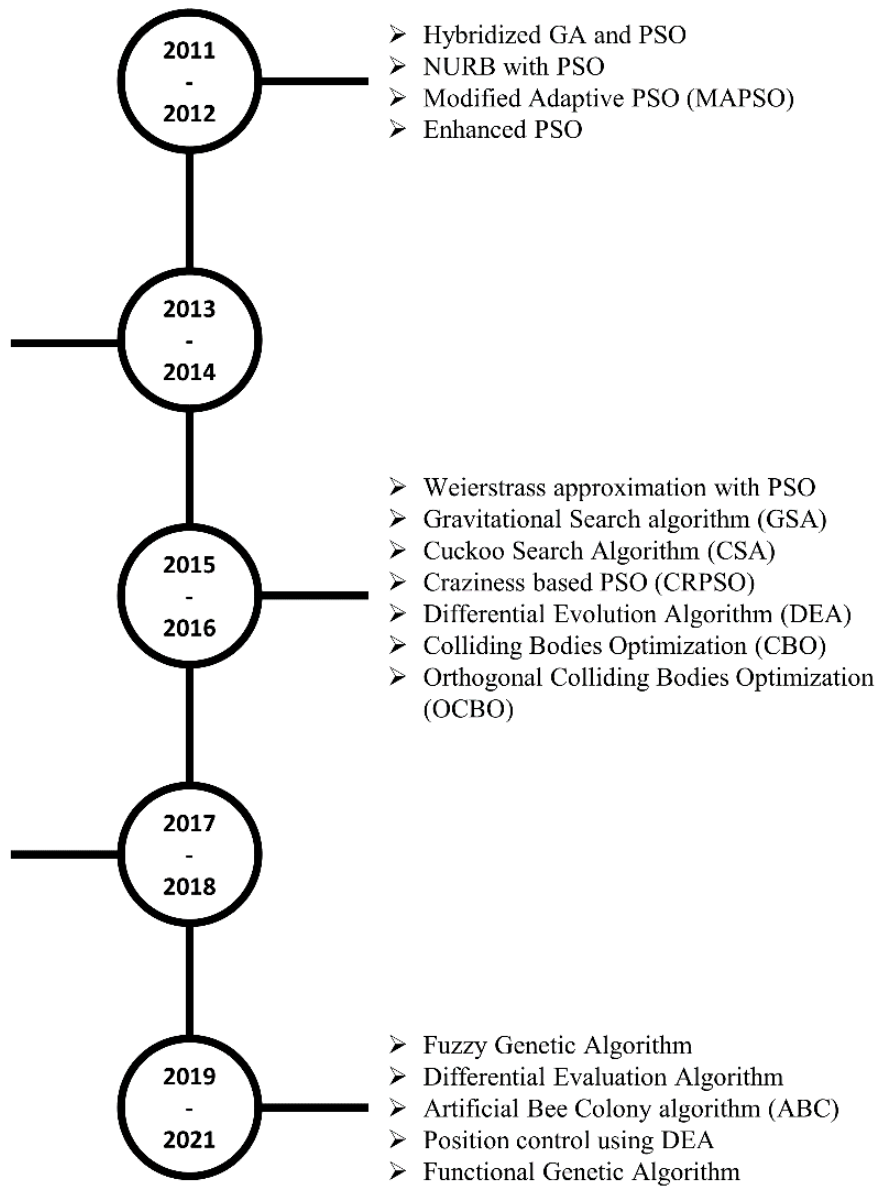

Figure 5. Timeline: Hammerstein model identification algorithms

\section{EXPERIMENTAL WORKS ON IDENTIFYING HAMMERSTEIN MODEL}

Empirical studies on nonlinear modelling and the identification of a real nonlinear plant are briefly discussed in this section. The experimental verification of such plants and processes is displayed in Table 1 . These process plants can be classified into several groups, such as chemical plants, mechanical plants, industrial plants and engineering plants such as amplified piezoelectric tube actuators [20], intelligent pneumatic actuator (IPA) [25], electrically stimulated muscle [31], ultrasonic motor [27], proton exchange membrane fuel cell (PEMFC) [28], turntable servo system [29], hydraulic mini excavator [26], twin rotor system (TRS) and flexible manipulator system (FMS) [72], and valve stiction [30].

Both the modelling and compensation of hysteresis nonlinearities of piezoelectric tube actuator using inverse rate-independent Prandtl-Ishlinskii model (RIPI) and inverse rate-dependent prandtl-ishlinskii model (RDPI), along with a controller [20]. For their identification, the Hammerstein model was applied. The IPA system that applied the Hammerstein model had been based on the RLS algorithm [25] and they use a discrete-time ARX model to represent the linear component of the Hammerstein model. The nonlinear Hammerstein model of the ultrasonic motor was reported in [27]. The PEMFC modelling that used the Hammerstein model [28] showed that the fractional Hammerstein PEMFC model with subspace identification algorithm not only avoided the analysis of internal complex mechanism but also accurately described the stack and nonlinear fractional characteristics. The identification of nonlinear predictive functional controller (NPFC) and nonlinear model, based on Hammerstein model for turntable servo system, was reported in [29]. A Hammerstein structure is used to represent the isometric response in the paper [31], which considers modelling of an electrically activated muscle, where they proposed the ARLS algorithm for identifying the parameters. Jui and Ahmad [72], a continuous-time Hammerstein model is developed based 
on AMVO-SCA and validated by modeling two real world plants such as TRS and FMS. In the Table 1, the objective function equation of the process plants with its parameter identification algorithm, number of design parameter from both nonlinear and linear subsystem of the Hammerstein model and the type of Hammerstein model also reported. From the tabular value it can clearly see that, most of the existing processes and plants identification algorithm identify an average number of design parameters and the majority of the previous study represent the model in discrete-time domain.

Table 1. Experimental verification table of Hammerstein model identification

\begin{tabular}{|c|c|c|c|c|c|c|}
\hline Plants and processes & $\begin{array}{l}\text { Parameter } \\
\text { estimation } \\
\text { algorithm }\end{array}$ & $\begin{array}{l}\text { Number } \\
\text { of design } \\
\text { parameter }\end{array}$ & $\begin{array}{c}\text { Types of } \\
\text { Hammerstein }\end{array}$ & Objective function equation & Year & Ref. \\
\hline Valve stiction & PSO & 8 & Discrete time & $\sum^{M}(y(k)-\hat{y}(k))^{2}$ & 2011 & {$[30]$} \\
\hline $\begin{array}{l}\text { Electrically stimulated } \\
\text { muscle }\end{array}$ & $\begin{array}{l}\text { Alternately } \\
\text { RLS (ARLS) }\end{array}$ & 5 & Discrete time & $\frac{1}{N-1} \sum_{t=1}^{N}\left(y_{t}-\bar{y}\right)^{2}$ & 2012 & [31] \\
\hline DC/DC boost converter & N/A & 8 & Discrete time & ${ }_{j}^{t=1} e^{2}(k)$ & 2015 & [24] \\
\hline $\begin{array}{l}\text { Amplified piezoelectric } \\
\text { actuators (APA) }\end{array}$ & RLS & 3 & N/A & N/A & 2016 & [20] \\
\hline Turntable servo system & $\begin{array}{l}\text { CLPSO \& } \\
\quad \text { PSO }\end{array}$ & 8 & Discrete time & $-\hat{y}(k)]^{2}$ & 2016 & [29] \\
\hline $\begin{array}{l}\text { Intelligent pneumatic } \\
\text { actuator (IPA) }\end{array}$ & RLS & 8 & Discrete time & $\left(1-\frac{|y-\hat{y}|}{y-\bar{y}}\right) * 100$ & 2017 & [25] \\
\hline Hydraulic mini excavator & N/A & N/A & Discrete time & $\min _{\bar{u} \in \mathbb{U}}\left\{\frac{1}{2} \bar{u}^{T} H \bar{u}+g^{T} \bar{u}+v\right\}$ & 2017 & [26] \\
\hline Ultrasonic motor (USM) & PSO and DE & 12 & N/A & $\sum_{i=1}^{q}\left[\sqrt{\sum_{k=1}^{h}\left[y_{i r}(k)-y_{i}(k)\right]^{2} / h}\right] / q$ & 2019 & [27] \\
\hline $\begin{array}{l}\text { Proton exchange } \\
\text { membrane fuel cell } \\
\text { (PEMFC) }\end{array}$ & FGA & 4 & Discrete time & $\min \sum_{i=1}^{N}\left[\left(\bar{V}_{i}-V_{i}\right)^{2}+\left(\bar{P}_{i}-P_{i}\right)^{2}\right]$ & 2019 & [28] \\
\hline $\begin{array}{l}\text { Twin rotor system (TRS) } \\
\text { and flexible manipulator } \\
\text { system (FMS) }\end{array}$ & AMVO-SCA & $5 \& 13$ & $\begin{array}{l}\text { Continuous } \\
\text { time }\end{array}$ & $\sum_{\eta=0}^{N}\left(\dot{y}\left(\eta t_{s}\right)-\bar{y}\left(\eta t_{s}\right)\right)^{2}$ & 2021 & [72] \\
\hline
\end{tabular}

\section{FUTURE RESEARCH TRENDS IN HAMMERSTEIN MODELS IDENTIFICATION}

This work surveyed several important metaheuristics algorithms for the identification of the Hammerstein model as they are described in different studies. Despite a limited theoretical foundation, the benefits of metaheuristics are vast. Nevertheless, several issues need to be highlighted to further determine their full potential. Metaheuristics assessments were mostly based on empirical comparisons. The following lists some significant related research issues that require further works in future.

- Despite the abundance of the existing literature on the Hammerstein model for system identification, the vast majority has focused on the SISO system. Nonetheless, works related to a multiple-input multipleoutput (MIMO) system is in scarcity. The real objects are always nonlinear in MIMO. In control practices, with the increasing requirements to deal with the complexities of modern technology, MIMO systems have more practical and wider applications than SISO systems do.

- Fractional order models have an important interest owing to their ability to represent many physical phenomena and being useful for modelling electrodynamics processes. These models have a remarkable advantage by representing the system with a low number of parameters. Identification of fractional order systems seems to be an active area within the research domain and mostly places focus on linear cases.

- Despite the presence of several algorithms in addressing several drawbacks, the future views hybridization as a powerful approach to combine the benefits of multiple algorithms. This hybridisation approach, nonetheless, is still in its trial-and-error phase although attempts can be noted as back as many years ago.

- It is worthwhile to investigate a few current metaheuristics algorithms that use a small number of coefficients to reduce the time spent determining the best setting for the coefficients. 
- $\quad$ Most earlier studies reflect discrete-time models, whereas most real-world systems are expressed in the continuous-time domain. It is worth researching a continuous-time Hammerstein model utilizing the metaheuristics method [73], [74].

\section{CONCLUSION}

The domain of system identification is related to control engineering that uses mathematical models of nonlinear systems based on input-output observations. Swarm intelligence and evolutionary computing are essential in this study area, whereby algorithms based on swarm intelligence have a role in nearly all science and engineering research areas. An extensive review is presented in this paper regarding the implementation of various metaheuristics algorithms for the identification of the Hammerstein model. This paper introduces the flow diagrams of common metaheuristics algorithms considering nonlinear Hammerstein system identification that may serve as guidance for those also in the artificial intelligence topic area. Some empirical studies on identifying the Hammerstein model are tabulated, including several uprising issues. It is hoped that more studies would investigate computations that involve nature and swarm intelligence to address a range of optimisation issues in actual implementations.

\section{ACKNOWLEDGEMENTS}

The Research and Innovation department of University Malaysia Pahang (UMP), and the Ministry of Higher Education Malaysia jointly financed this study through research grant RDU182303.

\section{REFERENCES}

[1] H. G. Natke, “Application of system identification in engineering,” Springer: Austria. 2014

[2] M. Schoukens and K. Tiels, "Identification of block-oriented nonlinear systems starting from linear approximations: A survey," Automatica, vol. 85, pp. 272-292, 2017, doi: 10.1016/j.automatica.2017.06.044

[3] S. Y. Wang, W. Y. Wang, L. J. Dang, and Y. X. Jiang, "Kernel Least Mean Square Based on the Nyström Method," Circuits, Systems, and Signal Processing, vol. 38, no. 7, pp. 3133-3151, 2019, doi: 10.1007/s00034-018-1006-2.

[4] Q. Shen and F. Ding, "Iterative estimation methods for Hammerstein controlled autoregressive moving average systems based on the key-term separation principle," Nonlinear Dynamics, vol. 75, no. 4, pp. 709-716, 2014, doi: 10.1007/s11071-013-1097-z.

[5] D. F. Wang D, D. Wang, and F. Ding, "Least squares based and gradient based iterative identification for Wiener nonlinear systems," Signal Processing, vol. 91, no. 5, pp. 1182-1189, 2011, doi: 10.1016/j.sigpro.2010.11.004.

[6] S. Cheng, Y. Wei, D. Sheng, and Y. Wang, "Identification for Hammerstein nonlinear systems based on universal spline fractional order LMS algorithm," Communications in Nonlinear Science and Numerical Simulation, vol. 79, p. 104901, 2019, doi: 10.1016/j.cnsns.2019.104901.

[7] E. Cuevas, J. Gálvez, S. Hinojosa, O. Avalos, D. Zaldívar, and M. Pérez-Cisneros, “A Comparison of Evolutionary Computation Techniques for IIR Model Identification,” Journal of Applied Mathematics, vol. 2014, pp. 1-9, 2014, doi: 10.1155/2014/827206

[8] S. H. Tsai and Y. W. Chen, "A Novel Fuzzy Identification Method Based on Ant Colony Optimization Algorithm," IEEE Access, vol. 4, pp. 3747-3756, 2016, doi: 10.1109/ACCESS.2016.2585670

[9] A. Alfi and M. M. Fateh, "Identification of nonlinear systems using modified particle swarm optimisation: A hydraulic suspension system," International Journal of Vehicle Mechanics and Mobility, vol. 49, no. 6, pp. 871-887, 2011, doi: $10.1080 / 00423114.2010 .497842$

[10] V. Rajinikanth and K. Latha, "Controller Parameter Optimization for Nonlinear Systems Using Enhanced Bacteria Foraging Algorithm,” Applied Computational Intelligence and Soft Computing, vol. 2012, pp. 1-12, 2012, doi: 10.1155/2012/214264.

[11] D. Cai, Y. Yu, and J. Wei, "A Modified Artificial Bee Colony Algorithm for Parameter Estimation of Fractional-Order Nonlinear Systems," IEEE Access, vol. 6, pp. 48600-48610, 2018, doi: 10.1109/ACCESS.2018.2859978.

[12] K. A. Mohideen, G. Saravanakumar, K. Valarmathi, D. Devaraj, and T. K. Radhakrishnan, "Real-coded Genetic Algorithm for system identification and tuning of a modified Model Reference Adaptive Controller for a hybrid tank system," Applied Mathematical Modelling, vol. 37, no. 6, pp. 3829-3847, 2013, doi: 10.1016/j.apm.2012.08.019.

[13] D. K. Khatod, V. Pant and J. Sharma, "Evolutionary programming based optimal placement of renewable distributed generators," in IEEE Transactions on Power Systems, vol. 28, no. 2, pp. 683-695, May 2013, doi: 10.1109/TPWRS.2012.2211044.

[14] A. H. Gandomi and A. H. Alavi, "Multi-stage genetic programming: A new strategy to nonlinear system modeling," Information Sciences, vol. 181, no. 23, pp. 5227-5239, 2011, doi: 10.1016/j.ins.2011.07.026.

[15] R. D. Al-Dabbagh, A. Kinsheel, S. Mekhilef, M. S. Baba, and S. Shamshirband, "System identification and control of robot manipulator based on fuzzy adaptive differential evolution algorithm," Advances in Engineering Software, vol. 78, pp. 60-66, 2014, doi: 10.1016/j.advengsoft.2014.08.009.

[16] J. J. Jui, M. H. Suid, Z. Musa, and M. A. Ahmad, "Identification of Liquid Slosh Behavior using Continuous-time Hammerstein Model based Sine Cosine Algorithm," in Proceedings of the 11th National Technical Seminar on Unmanned System Technology 2019, 2020, pp. 345-356, doi: 10.1007/978-981-15-5281-6_24.

[17] J. J. Jui, M. H. Suid, M. R. Ghazali, M. A. Ahmad, and M. Z. M. Tumari, "Modified Sine Cosine Algorithm for Identification of Liquid Slosh based on Continuous-time Hammerstein Model," Journal of Physics: Conference Series, Volume 1529, The 2nd Joint International Conference on Emerging Computing Technology and Sports (JICETS) 2019, Bandung, Indonesia, 25-27 November 2019, vol. 1529, no. 4, 2020, doi: 10.1088/1742-6596/1529/4/042090.

[18] J. J. Jui, M. A. Ahmad, M. S. M. Ali, M. A. Zawawi, and M. F. M. Jusof, "Identification of the Thermoelectric Cooler Using Hybrid Multi-Verse Optimizer and Sine Cosine Algorithm Based Continuous-Time Hammerstein Model," Cybernetics and Information Technologies, vol. 666, no. 3, pp. 345-356, 2021, doi: 10.2478/cait-2021-0036. 
[19] J. J. Jui, M. A. Ahmad, M. S. M. Ali, M. A. Zawawi, and M. F. M. Jusof, "Thermoelectric cooler identification based on continuous-time Hammerstein model using metaheuristics algorithm," in 2021 International Conference on Software Engineering \& Computer Systems and 4th International Conference on Computational Science and Information Management (ICSECSICOCSIM), 2021, pp. 556-561, doi: 10.1109/ICSECS52883.2021.00108.

[20] O. Aljanaideh, D. Habineza, M. Rakotondrabe, and M. Al Janaideh, "Experimental comparison of rate-dependent hysteresis models in characterizing and compensating hysteresis of piezoelectric tube actuators," Physica B: Condensed Matter, vol. 486, pp. 64-68, 2016, doi: 10.1016/j.physb.2015.10.021.

[21] O. Ait Maatallah, A. Achuthan, K. Janoyan, and P. Marzocca, "Recursive wind speed forecasting based on Hammerstein AutoRegressive model," Applied Energy, vol. 145, pp. 191-197, 2015, doi: 10.1016/j.apenergy.2015.02.032.

[22] Y. Guo, J. Mao and K. Zhou, "Rate-Dependent Modeling and Hœ Robust Control of GMA Based on Hammerstein Model With Preisach Operator," in IEEE Transactions on Control Systems Technology, vol. 23, no. 6, pp. 2432-2439, Nov. 2015, doi: 10.1109/TCST.2015.2413497.

[23] J. Zhao, X. Ma, S. Zhao, and J. Fei, "Hammerstein identification of supercharged boiler superheated steam pressure using Laguerre-Fuzzy model," International Journal of Heat and Mass Transfer, vol. 70, pp. 33-39, 2014, doi: 10.1016/j.ijheatmasstransfer.2013.10.056.

[24] F. Alonge, R. Rabbeni, M. Pucci and G. Vitale, "Identification and Robust Control of a Quadratic DC/DC Boost Converter by Hammerstein Model," in IEEE Transactions on Industry Applications, vol. 51, no. 5, pp. 3975-3985, Sept.-Oct. 2015, doi: 10.1109/TIA.2015.2416154.

[25] S. F. Sulaiman, M. F. Rahmat, A. A. M. Faudzi, K. Osman, N. H. Sunar, and S. N. S. Salim, "Hammerstein model based RLS algorithm for modeling the intelligent Pneumatic Actuator (IPA) system," International Journal on Advanced Science, Engineering and Information Technology, vol. 7, no. 4, pp. 1457-1463, 2017, doi: 10.18517/ijaseit.7.4.3149.

[26] F. A. Bender, S. Göltz, T. Bräunl and O. Sawodny, "Modeling and Offset-Free Model Predictive Control of a Hydraulic Mini Excavator," in IEEE Transactions on Automation Science and Engineering, vol. 14, no. 4, pp. 1682-1694, Oct. 2017, doi: 10.1109/TASE.2017.2700407.

[27] S. Lu and S. Jingzhuo, "Nonlinear Hammerstein model of ultrasonic motor for position control using differential evolution algorithm," Ultrasonics, vol. 94, no. December, pp. 20-27, 2019, doi: 10.1016/j.ultras.2018.12.012.

[28] Z. Qi, Q. Sun, W. Ge, and Y. He, "Nonlinear modeling of PEMFC based on fractional order subspace identification," Asian Journal of Control, vol. 22, pp. 1892-1900, 2019, doi: 10.1002/asjc.2092.

[29] Q. Zhang, Q. Wang, and G. Li, "Nonlinear modeling and predictive functional control of Hammerstein system with application to the turntable servo system," Mechanical Systems and Signal Processing, vol. 72-73, pp. 383-394, 2016, doi: 10.1016/j.ymssp.2015.09.011.

[30] S. Sivagamasundari and D. Sivakumar, "Estimation of valve stiction using particle swarm optimization," Sensors and Transducers, vol. 129, no. 6, pp. 149-162, 2011.

[31] F. Le, I. Markovsky, C. T. Freeman, and E. Rogers, "Recursive identification of Hammerstein systems with application to electrically stimulated muscle," Control Engineering Practice, vol. 20, no. 4, pp. 386-396, 2012, doi: 10.1016/j.conengprac.2011.08.001.

[32] D. Wang, L. Mao, and F. Ding, "Recasted models-based hierarchical extended stochastic gradient method for MIMO nonlinear systems," IET Control Theory \& Applications, vol. 11, no. 4, pp. 476-485, 2017, doi: 10.1049/iet-cta.2016.0017.

[33] N. I. Chaudhary, M. A. Manzar, and M. A. Z. Raja, "Fractional Volterra LMS algorithm with application to Hammerstein control autoregressive model identification," Neural Computing and Applications, vol. 31, no. 9, pp. 5227-5240, 2019, doi: 10.1007/s00521018-3362-z.

[34] J. Hou, T. Liu, B. Wahlberg, and M. Jansson, "Subspace Hammerstein Model Identification under Periodic Disturbance," IFACPapersOnLine, vol. 51, no. 15, pp. 335-340, 2018, doi: 10.1016/j.ifacol.2018.09.157.

[35] J. Ma, B. Huang and F. Ding, "Iterative Identification of Hammerstein Parameter Varying Systems With Parameter Uncertainties Based on the Variational Bayesian Approach," in IEEE Transactions on Systems, Man, and Cybernetics: Systems, vol. 50, no. 3, pp. 1035-1045, March 2020, doi: 10.1109/TSMC.2017.2756913.

[36] A. Akramizadeh, A. A. Farjami and H. Khaloozadeh, "Nonlinear Hammerstein model identification using genetic algorithm," Proceedings 2002 IEEE International Conference on Artificial Intelligence Systems (ICAIS 2002), 2002, pp. 351-356, doi: 10.1109/ICAIS.2002.1048126.

[37] Z. L. Lai, X. D. Liu, and J. Geng, "Hammerstein-based model of piezoceramic actuator and its identification," Guangxue Jingmi Gongcheng(Optics and Precision Engineering), vol. 20, no. 9, pp. 2087-2094, 2012, doi: 10.3788/OPE.20122009.2087.

[38] W. J. Li, S. J. Lin, and X. Wang, "New method for identification of Hammerstein model," Acta Metrologica Sinica, vol. 36, no. 4, pp. 418-422, 2015.

[39] H. Da Song, P. Zhou, H. Wang, and T. Y. Chai, "Nonlinear subspace modeling of multivariate molten iron quality in blast furnace ironmaking and its application," Zidonghua Xuebao/Acta Autom. Sin., vol. 42, no. 11, pp. 1664-1679, 2016.

[40] M. J. Moghaddam, H. Mojallali and M. Teshnehlab, "A multiple-input-single-output fractional-order Hammerstein model identification based on modified neural network," Mathematical Methods in the Applied Sciences, vol. 41, no. 16, pp. 6252-6271, 2018, doi: 10.1002/mma.5136.

[41] M. A. Z. Raja, A. A. Shah, A. Mehmood, N. I. Chaudhary, and M. S. Aslam, "Bio-inspired computational heuristics for parameter estimation of nonlinear Hammerstein controlled autoregressive system," Neural Computing and Applications, vol. 29, no. 12, pp. 1455-1474, 2018, doi: 10.1007/s00521-016-2677-x.

[42] M. Jahani Moghaddam, H. Mojallali, and M. Teshnehlab, "Recursive identification of multiple-input single-output fractionalorder Hammerstein model with time delay," Applied Soft Computing, vol. 70, pp. 486-500, 2018, doi: 10.1016/j.asoc.2018.05.046.

[43] A. Mehmood, N. I. Chaudhary, A. Zameer, and M. A. Z. Raja, "Novel computing paradigms for parameter estimation in Hammerstein controlled auto regressive auto regressive moving average systems," Applied Soft Computing, vol. 80, no. 5, pp. 263-284, 2019, doi: 10.1016/j.asoc.2019.03.052.

[44] A. Mehmood, N. I. Chaudhary, A. Zameer, and M. A. Z. Raja, "Backtracking search optimization heuristics for nonlinear Hammerstein controlled auto regressive auto regressive systems," ISA transactions, vol. 91, pp. 99-113, 2019, doi: 10.1016/j.isatra.2019.01.042.

[45] H. Fan and W. Lin, "Parameter estimation of the MISO nonlinear system based on improved particle swarm optimization," Applied Mechanics and Materials, vol. 130-134, pp. 2563-2567, 2012, doi: 10.4028/www.scientific.net/AMM.130-134.2563.

[46] X. Xu, F. Wang, G. Liu, and F. Qian, "Identification of Hammerstein systems using key-term separation principle, auxiliary model and improved particle swarm optimisation algorithm," IET Signal Processing, vol. 7, no. 8, pp. 766-773, 2013, doi: 10.1049/iet-spr.2013.0042. 
[47] X. Hong and S. Chen, "The system identification and control of Hammerstein system using non-uniform rational B-spline neural network and particle swarm optimization," Neurocomputing, vol. 82, pp. 216-223, 2012, doi: 10.1016/j.neucom.2011.11.016.

[48] Er-Wei Bai and Minyue Fu, "A blind approach to Hammerstein model identification," in IEEE Transactions on Signal Processing, vol. 50, no. 7, pp. 1610-1619, July 2002, doi: 10.1109/TSP.2002.1011202.

[49] J. Sun and X. Liu, "A novel APSO-aided maximum likelihood identification method for Hammerstein systems," Nonlinear Dynamics, vol. 73, no. 1-2, pp. 449-462, 2013, doi: 10.1007/s11071-013-0800-4.

[50] Zhangqian, W. Qunjing and L. Guoli, "Evolutionary identification algorithm for Hammerstein system and its convergence analysis," 2014 9th IEEE Conference on Industrial Electronics and Applications, 2014, pp. 2122-2126, doi: 10.1109/ICIEA.2014.6931522.

[51] A. Kaveh and V. R. Mahdavi, "Colliding bodies optimization: A novel meta-heuristic method," Computers \& Structures, vol. 139, pp. 18-27, 2014, doi: 10.1016/j.compstruc.2014.04.005.

[52] A. Panda and S. Pani, "A new model based on Colliding Bodies Optimization for identification of Hammerstein plant," 2014 Annual IEEE India Conference (INDICON), 2014, pp. 1-5, doi: 10.1109/INDICON.2014.7030381.

[53] P. S. Pal, R. Kar, D. Mandal, and S. P. Ghoshal, "An efficient identification approach for stable and unstable nonlinear systems using Colliding Bodies Optimization algorithm," ISA transactions, vol. 59, pp. 85-104, 2015, doi: 10.1016/j.isatra.2015.08.012.

[54] A. Gotmare, R. Patidar, and N. V. George, "Nonlinear system identification using a cuckoo search optimized adaptive Hammerstein model," Expert systems with applications, vol. 42, no. 5, pp. 2538-2546, 2015, doi: 10.1016/j.eswa.2014.10.040.

[55] X. Xu, Q. Zhu, F. Wang, F. Qian, and F. Dai, "Estimation of System Models by Swarm Intelligent Method," Sensors \& Transducers, vol. 183, no. 12, pp. 293-299, 2014.

[56] G. Liu, X. Xu, and F. Wang, "Modeling of a class of nonlinear dynamic system," Sensors and Transducers, vol. 169, no. 4, pp. $153-158,2014$

[57] P. S. Pal, S. Banerjee, R. Kar, D. Mandal and S. P. Ghoshal, "Parametric identification of Box-Jenkins structured closed-loop Hammerstein systems using gravitational search algorithm," 2015 International Conference on Science and Technology (TICST), 2015, pp. 243-247, doi: 10.1109/TICST.2015.7369365.

[58] Ş. Özer, H. Zorlu and S. Mete, "A comparison study of system identification using Hammerstein model," 2015 11th International Conference on Innovations in Information Technology (IIT), 2015, pp. 367-372, doi: 10.1109/INNOVATIONS.2015.7381569.

[59] J. Chen and X. Wang, "Identification of Hammerstein systems with continuous nonlinearity," Information Processing Letters, vol. 115, no. 11, pp. 822-827, 2015, doi: 10.1016/j.ipl.2015.06.004.

[60] K. Hammar, T. Djamah and M. Bettayeb, "Fractional hammerstein system identification using particle swarm optimization," 2015 7th International Conference on Modelling, Identification and Control (ICMIC), 2015, pp. 1-6, doi: 10.1109/ICMIC.2015.7409483.

[61] P. S. Pal et al., "Hammerstein model based system identification using craziness based particle swarm optimization algorithm," 2016 International Conference on Communication and Signal Processing (ICCSP), 2016, pp. 1623-1627, doi: 10.1109/ICCSP.2016.7754436

[62] L. Ma and X. Liu, "A novel APSO-aided weighted LSSVM method for nonlinear hammerstein system identification," Journal of the Franklin Institute, vol. 354, no. 4, pp. 1892-1906, 2017, doi: 10.1016/j.jfranklin.2016.12.022.

[63] S. Boubaker, "Identification of nonlinear Hammerstein system using mixed integer-real coded particle swarm optimization: application to the electric daily peak-load forecasting," Nonlinear Dynamics, vol. 90, no. 2, pp. 797-814, 2017, doi: 10.1007/s11071-017-3693-9.

[64] P. Jangir, S. A. Parmar, I. N. Trivedi, and R. H. Bhesdadiya, "A novel hybrid Particle Swarm Optimizer with multi verse optimizer for global numerical optimization and Optimal Reactive Power Dispatch problem," Engineering Science and Technology, an International Journal, vol. 20, no. 2, pp. 570-586, 2017, doi: 10.1016/j.jestch.2016.10.007.

[65] W. Xiong, M. Chen, X. Wang, and B. Xu, "System identification method for Hammerstein model based on improved differential evolution algorithm," J. Nanjing Univ. Sci. Technol., vol. 37, no. 4, pp. 536-542, 2015.

[66] S. Mete, S. Ozer, and H. Zorlu, "System identification using Hammerstein model optimized with differential evolution algorithm," AEU - Int. J. Electron. Commun., vol. 70, no. 12, pp. 1667-1675, 2016, doi: 10.1016/j.aeue.2016.10.005.

[67] J. Vesterstrom and R. Thomsen, "A comparative study of differential evolution, particle swarm optimization, and evolutionary algorithms on numerical benchmark problems," Proceedings of the 2004 Congress on Evolutionary Computation (IEEE Cat. No.04TH8753), 2004, pp. 1980-1987 Vol.2, doi: 10.1109/CEC.2004.1331139.

[68] A. Mehmood, A. Zameer, N. I. Chaudhary, S. H. Ling, and M. A. Z. Raja, "Design of meta-heuristic computing paradigms for Hammerstein identification systems in electrically stimulated muscle models," Neural Comput. Appl., vol. 32, no. 16, pp. 1246912497, 2020, doi: 10.1007/s00521-020-04701-4.

[69] H. Wenwen and S. Jingzhuo, "MISO Nonlinear Gauss-Hammerstein Model Identification of Ultrasonic Motor," ournal of Control, Automation and Electrical Systems, vol. 32, pp. 356-366, 2021, doi: 10.1007/s40313-020-00676-8.

[70] A. Panda and S. Pani, "A WNN model trained with orthogonal colliding bodies optimization for accurate identification of hammerstein plant," 2016 IEEE Congress on Evolutionary Computation (CEC), 2016, pp. 1100-1106, doi: 10.1109/CEC.2016.7743911.

[71] E. Cuevas et al., "Nonlinear system identification based on ANFIS-Hammerstein model using Gravitational search algorithm," Applied Intelligence, vol. 48, no. 1, pp. 182-203, 2018, doi: 10.1007/s10489-017-0969-1.

[72] J. J. Jui and M. A. Ahmad, "A hybrid metaheuristic algorithm for identification of continuous-time Hammerstein systems," Applied Mathematical Modelling, vol. 95, pp. 339-360, 2021, doi: 10.1016/j.apm.2021.01.023.

[73] M. A. Ahmad, S. I. Azuma, and T. Sugie, "Identification of continuous-time Hammerstein systems by simultaneous perturbation stochastic approximation," Expert Systems with Applications, vol. 43, pp. 51-58, Jan. 2016, doi: 0.1016/j.eswa.2015.08.041.

[74] M. A. Ahmad, Z. Musa, M. H. Suid, and M. Z. M. Tumari, "Grey wolf optimizer for identification of liquid slosh behavior using continuous-time hammerstein model," Bulletin of Electrical Engineering and Informatics, vol. 9, no. 2, pp. 542-549, 2020, doi: 10.11591/eei.v9i2.2074. 


\section{BIOGRAPHIES OF AUTHORS}
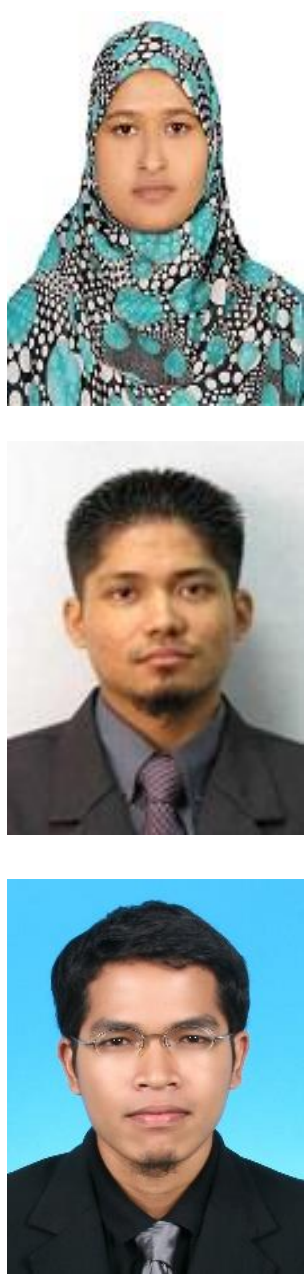

Julakha Jahan Jui (D) SO SC Pas completed her B.Sc. in Computer Science and Engineering from Pabna University of Science and Technology, Bangladesh in 2017 and received her M.Sc. in Electrical and Electronics Engineering from University Malaysia Pahang, Malaysia. Her main areas of interests are metaheuristics, machine learning and artificial intelligence. She can be contacted at email: julakha.ump@gmail.com.

Mohd Ashraf Ahmad (DD 81 SC P received his first degree in B.Eng. Electrical Mechatronics and Master Degree in M.Eng. Mechatronics and Automatic Control from University of Technology Malaysia (UTM) in 2006 and 2008, respectively. In 2015, he received a Ph.D. in Informatics (Systems Science) from Kyoto University. Currently, he is an Associate Professor in Faculty of Electrical and Electronics Engineering, University Malaysia Pahang (UMP). His current research interests are model-free control, control of mechatronic systems, nonlinear system identification and vibration control. He can be contacted at email: mashraf@ump.edu.my

Muhammad Ikram bin Mohd Rashid (D) 8d SC P received his Bachelor Electrical Power Engineering in 2008 and Master Electrical Power Engineering in 2010 from University Technology Malaysia. In 2010, he joined the Faculty of Electrical \& Electronics Engineering, University Malaysia Pahang, as a Lecturer. Muhammad Ikram is a member of the IEEE Malaysia, Institute of Electrical Engineers of Malaysia and the Society of Electrical Engineers of Malaysia. His research interest includes power electronics, electric vehicle, energy conversions, semiconductor devices and renewable energy. He can be contacted at email: mikram@ump.edu.my. 